01.3

\title{
Оценка времени предсказуемости зашумленной хаотической динамики по точечным последовательностям
}

\author{
(C) Я.Х. Мохаммад ${ }^{1}$, О.Н. Павлова ${ }^{1}$, А.Н. Павлов ${ }^{1,2, \text { Ф }}$ \\ ${ }^{1}$ Саратовский государственный университет им. Н.Г. Чернышевского \\ ${ }^{2}$ Саратовский государственный технический университет \\ им. Гагарина Ю.А. \\ ฯ E-mail: pavlov.alexeyn@gmail.com
}

Поступило в Редакцию 29 марта 2016 г.

Предложен метод повышения точности расчета времени предсказуемости зашумленной хаотической динамики по точечным последовательностям. На примере режима фазово-когерентного хаоса проиллюстрированы общие закономерности, наблюдаемые при применении данного метода к точечным последовательностям двух типов моделей пороговых устройств.

DOI: $10.21883 /$ PJTF.2017.02.44186.16268

Основная особенность систем, демонстрирующих хаотические режимы колебаний, состоит в том, что малое изменение начальных условий или параметров системы приводит к потере предсказуемости колебательного процесса через некоторое время. Степень предсказуемости будет меняться, если хаотический режим исследуется в условиях дополнительного присутствия различных источников флуктуаций. Чтобы определить максимальное время, в течение которого можно осуществить прогноз поведения системы, было введено понятие горизонта предсказуемости [1], который зависит от наличия априорных сведений об исследуемой системе и статистики присутствующего шума. Если прогноз осуществляется на основе экспериментальных данных, то в качестве времени предсказуемости $\tau_{p}$ часто используют „время Ляпунова“, т.е. время, за которое расстояние между соседними фазовыми траекториями системы возрастает в $e$ раз. Оценка предела предсказуемости системы с хаотической динамикой как величины, обратной старшему показателю Ляпунова $\left(\tau_{p}=1 / \lambda_{1}\right)$, означает, что если 
расчеты старшего показателя приведут к неправильному значению $\lambda_{1}$, то возникнет ошибка в определении времени прогноза.

Точность расчета $\lambda_{1}$ зависит от того, какая информация о динамике системы известна. Если заданы уравнения математической модели, то вычислить спектр ляпуновских показателей можно с требуемой точностью, применяя стандартный алгоритм [2]. Если анализ проводится по временной зависимости одной из динамических переменных, то применяются методы реконструкции динамических систем [3-5]. Задача расчета показателей Ляпунова усложняется, если эта переменная подвергается преобразованиям, в результате которых сокращается объем доступной информации о динамике. В частности, в работах [6-9] рассмотрены преобразования хаотических сигналов пороговыми устройствами, в результате которых носителями информации о динамике системы становятся последовательности стереотипных импульсов, генерируемых при превышении порогового уровня, классифицируемые как точечные последовательности.

Возможность оценки по точечным последовательностям таких характеристик хаотической динамики, как корреляционная размерность и показатели Ляпунова, ранее была рассмотрена в работах [6-13], где сформулированы условия, при которых можно правильно идентифицировать режим динамики на входе порогового устройства. При высокой частоте генерации импульсов пороговым устройством была доказана теорема Зауэра, применимая к точечным последовательностям моделей типа „накопление-сброс“ (НС) [10]. При сравнительно низкой частоте генерации, а также для других моделей пороговых устройств, например моделей типа „пересечения порога“ (ПП), возможность расчета показателей Ляпунова была подтверждена численными расчетами [11-15]. Однако в отмеченных публикациях не учитывалось наличие измерительного шума во входном колебательном процессе, приводящего к варьированию длительности межимпульсных интервалов (МИ) на выходе порогового устройства. Целью данной работы является модификация метода расчета старшего показателя Ляпунова [5], которая позволяет повысить точность проводимых оценок времени предсказуемости хаотической динамики в случае, когда точечная последовательность может быть зашумленной, но при этом отсутствует информация об интенсивности шума.

Основная идея предложенного подхода состоит в следующем. Стандартный алгоритм [5] предусматривает расчет $\lambda_{1}$ как средней скорости экспоненциального разбегания траекторий в реконструированном

Письма в ЖТФ, 2017, том 43, вып. 2 
фазовом пространстве. При анализе одномерных проекций фазовых траекторий, принадлежащих хаотическому аттрактору, после проведения реконструкции определяются границы линейного приближения $\left[l_{\min }, l_{\max }\right]$ для вектора возмущения $r(t)$, соответствующие разбеганию траекторий, обусловленному динамикой системы. При выходе за верхнюю границу $l_{\max }$ происходит недооценка $\lambda_{1}$ из-за нелинейного ограничения размера вектора возмущения. Нижняя граница вводится для исключения эффекта дополнительного разбегания траекторий, вызванного присутствием измерительного шума в анализируемом сигнале. Алгоритм [5] применим и при анализе точечных последовательностей после их предварительной обработки. В случае НС-модели, описывающей процесс генерации импульсов в моменты времени $T_{i}$ при достижении интегралом от входного сигнала $S(t)$ порогового уровня $\theta$, проводится восстановление входного сигнала $S\left(T_{i}\right) \approx \theta /\left(T_{i+1}-T_{i}\right)$, точность которого возрастает с увеличением частоты генерации [7]. Для ПП-модели, предусматривающей генерацию импульсов при переходе входного сигнала через пороговый уровень, используется прием, основанный на аппроксимации усредненной мгновенной частоты колебаний $\omega\left(T_{i}\right)=2 \pi /\left(T_{i+1}-T_{i}\right)$ [11]. Далее осуществляется переход к сигналу с равномерной выборкой (соответственно $S(i \Delta t)$ и $\omega(i \Delta t)$ ) путем интерполяции полученных отсчетов, и этот сигнал анализируется на основе метода [5].

Наличие шума создает ряд сложностей при проведении вычислений. Чтобы повысить точность расчета старшего показателя Ляпунова, предлагаем проводить анализ зависимости $\lambda_{1}$ от максимальной ошибки ориентации $\alpha$, которая определяется как угол между векторами возмущения до и после перенормировки. Из общих соображений можно предположить, что очень малые и очень большие $\alpha$ будут приводить к снижению величины $\lambda_{1}$, поэтому при расчете показателя Ляпунова необходимо задавать промежуточные значения.

На рисунке, $a$ приведены результаты расчета старшего показателя Ляпунова режима фазово-когерентного хаоса в модели Ресслера

$$
\begin{aligned}
& \frac{d x}{d t}=-y-z, \quad \frac{d y}{d t}=x+a y, \quad \frac{d z}{d t}=b+z(x-c), \\
& a=0.15, \quad b=0.2, \quad c=10
\end{aligned}
$$

по последовательности 2000 МИ на выходе НС-модели, на вход которой поступал сигнал $S(t)=x(t)+C+D \xi(t)$, где $C-$ постоянная величина,

Письма в ЖТФ, 2017, том 43, вып. 2 

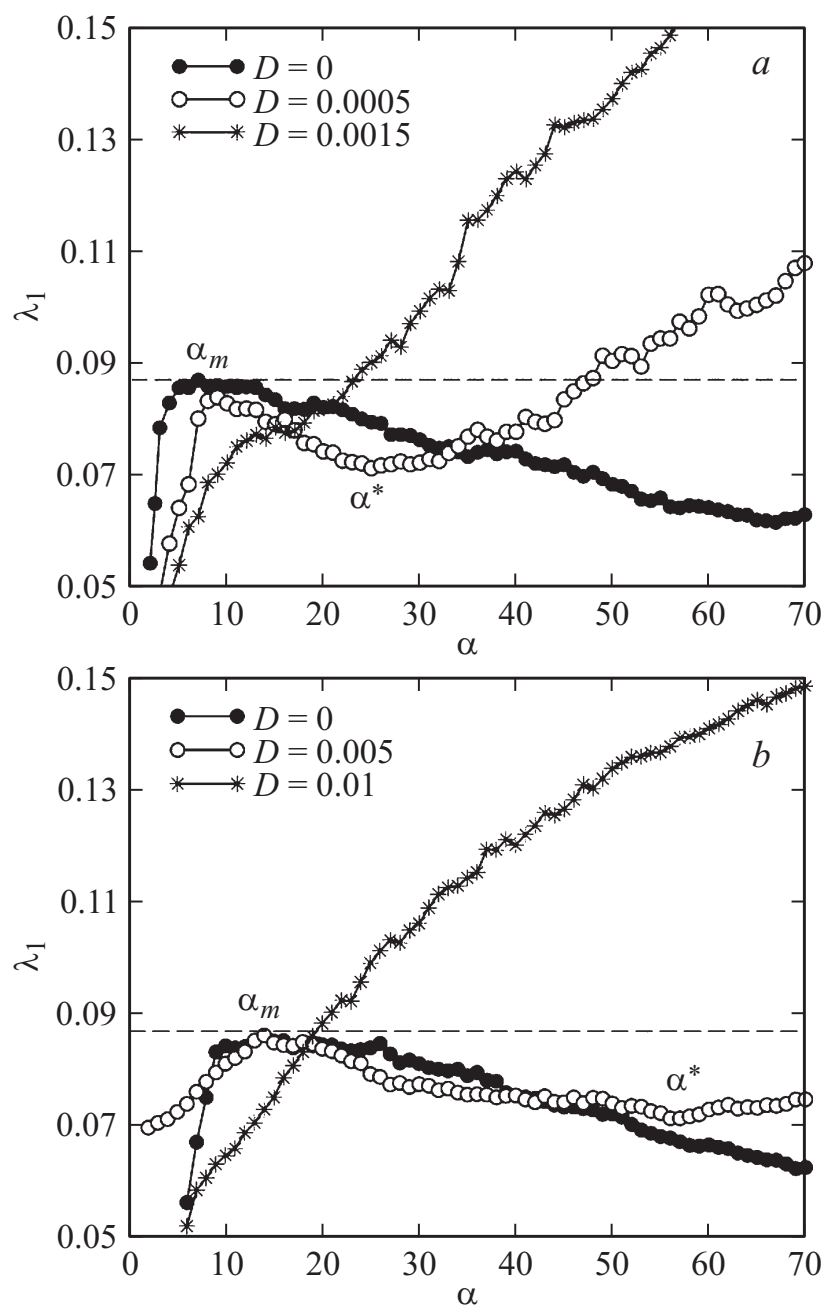

Зависимости старшего показателя Ляпунова хаотического режима колебаний системы (1), вычисленные по последовательностям МИ модели $\mathrm{HC}(a)$ и ПП $(b)$, от максимальной ошибки ориентации вектора возмущения (в градусах). 
вводимая для того, чтобы избежать отрицательных значений входного сигнала (в проводимых расчетах $C=35), \xi(t)$ - белый шум. Представленные результаты получены при значениях $l_{\min }=0.01, l_{\max }=0.1$. Для удобства проводимых вычислений предварительно осуществлялось приведение сигнала $S(i \Delta t)$ к единичному интервалу.

Отправными в исследованиях являются зависимости $\lambda_{1}(\alpha)$, относящиеся к динамике в отсутствие шума $(D=0)$. В этом случае наблюдается максимум $\lambda_{1}(\alpha)$ при $\alpha=\alpha_{m}$, который соответствует теоретически ожидаемому значению старшего показателя, вычисленному по известным уравнениям математической модели (1) с применением алгоритма [2] (в рассматриваемом примере $\lambda_{1}=0.087-$ пунктирная линия на рисунке, $a$ ). Слева от максимума недооценка $\lambda_{1}$ происходит из-за низкой вероятности выбора малых по модулю векторов возмущения и частого выхода за границы линейного приближения. Справа от максимума из-за возрастающих ошибок ориентации приходится учитывать разбегание траекторий в направлениях, ортогональных направлению их максимального разбегания.

Важно отметить, что приведенная зависимость $\lambda_{1}(\alpha)$ имеет характерный вид, качественно не меняющийся при анализе систем с различным хаотическим поведением (дополнительно были исследованы режимы хаотических колебаний в модели Лоренца, генераторе с инерционной нелинейностью, а также рассмотрены случаи слабого и развитого хаоса в модели Ресслера). Оценка времени предсказуемости с помощью рисунка, $a$ приводит к значению $\tau_{p}\left(\alpha_{m}\right)=1 / \lambda_{1}\left(\alpha_{m}\right)$. Отклонение от $\alpha_{m}$ означает, что вычисленная величина $\tau_{p}$ будет существенно превышать длительность прогноза, вычисленную по уравнениям системы (1), приводя к недостоверным выводам о степени детерминированности анализируемой динамики.

Характер зависимости $\lambda_{1}(\alpha)$ меняется при наличии шума в последовательности МИ, который может быть вызван присутствием помех во входном сигнале или флуктуирующим пороговым уровнем. При достижении значения $\alpha^{*}$ появляется положительный наклон $\lambda_{1}(\alpha)$ в области $\alpha>\alpha^{*}$, и соответствующее значение $\alpha^{*}$ стремится к $\alpha_{m}$ с ростом интенсивности шума. Такие изменения зависимости $\lambda_{1}(\alpha)$ также являются характерными для всех рассмотренных примеров систем с хаотической динамикой при наличии шума в последовательности МИ. Обнаруженная закономерность позволяет по виду зависимости $\lambda_{1}(\alpha)$ качественно судить о присутствии шума и его интенсивности, так как

4 Письма в ЖТФ, 2017, том 43, вып. 2 
с увеличением $D$ не только уменьшается значение $\alpha^{*}$, но и растет наклон $\lambda_{1}(\alpha)$ в области $\alpha>\alpha^{*}$. Начиная с некоторой интенсивности $D$, максимум зависимости $\lambda_{1}(\alpha)$ исчезает, и результаты оценки времени предсказуемости перестают быть достоверными. Для примера, приведенного на рисунке, $a$, результаты расчетов становятся недостоверными при $D=0.0015$, что соответствует отношению интенсивности шума к средней длительности МИ $5 \cdot 10^{-4}$.

В случае ПП-модели были обнаружены аналогичные закономерности. На рисунке, $b$ приведены примеры проведенных расчетов для режима фазово-когерентного хаоса в системе (1) в отсутствие шума и при наличии флуктуаций в последовательности МИ. В качестве входного сигнала ПП-модели была рассмотрена переменная состояния $x(t)$, а порог определялся равенством $\theta=0$. По-прежнему в отсутствие шума наблюдается характерный максимум $\lambda_{1}(\alpha)$ (минимум $\tau_{p}(\alpha)$ ), соответствующий теоретически ожидаемым оценкам (пунктирная линия), а при наличии шума происходят изменения наклона в области $\alpha>\alpha^{*}$, и величина наклона (как и соответствующее значение $\alpha^{*}$ ) меняется с ростом интенсивности шума.

Таким образом, в данной работе предложена модификация метода расчета старшего показателя Ляпунова, предусматривающая построение зависимости величины показателя от ошибки ориентации векторов в реконструированном фазовом пространстве. Предложенный подход позволил установить характерное поведение $\lambda_{1}(\alpha)$, по виду которого можно сделать вывод о присутствии шума и при наличии данных, полученных при разном уровне шума, провести их качественное сравнение по интенсивности присутствующих помех. Выбор параметра $\alpha$, соответствующего максимуму $\lambda_{1}(\alpha)$, позволяет повысить точность оценки времени предсказуемости при анализе различных типов зашумленных точечных последовательностей.

Исследование выполнено при поддержке гранта Российского научного фонда (проект № 14-12-00224).

\section{Список литературы}

[1] Lighthill J. // Proc. Roy. Soc. Ser. A. 1986. V. 407. P. 35.

[2] Benettin G., Galgani L., Giorgilli A., Strelcyn J.M. // Meccanica. 1980. V. 15. P. 9. 
[3] Takens F. // Dynamical Systems and Turbulence / Eds D.A. Rang and L.S. Young. Lecture Notes in Mathematics. V. 898. Berlin: Springer-Verlag, 1981. P. 366.

[4] Packard N.H., Crutchfield J.P., Farmer J.D., Shaw R.S. // Phys. Rev. Lett. 1980. V. 45. P. 712.

[5] Wolf A., Swift J.B., Swinney H.L., Vastano J.A. // Physica D. 1985. V. 16. P. 285.

[6] Sauer T. // Chaos. 1995. V. 5. P. 127.

[7] Racicot D.M., Longtin A. // Physica D. 1997. V. 104. P. 184.

[8] Hegger R., Kantz H. // Europhys. Lett. 1997. V. 38. P. 267.

[9] Castro R., Sauer T. // Phys. Rev. E. 1997. V. 55. P. 287.

[10] Sauer T. // Phys. Rev. Lett. 1994. V. 72. P. 3911.

[11] Janson N.B., Pavlov A.N., Neiman A.B., Anishchenko V.S. // Phys. Rev. E. 1998. V. 58. P. R4.

[12] Pavlov A.N., Sosnovtseva O.V., Mosekilde E., Anishchenko V.S. // Phys. Rev. E. 2000. V. 61. P. 5033.

[13] Pavlov A.N., Sosnovtseva O.V., Mosekilde E., Anishchenko V.S. // Phys. Rev. E. 2001. V. 63. P. 036205.

[14] Pavlov A.N., Pavlova O.N., Mohammad Y.K., Kurths J. // Phys. Rev. E. 2015. V. 91. P. 022921.

[15] Pavlov A.N., Pavlova O.N., Mohammad Y.K., Kurths J. // Chaos. 2015. V. 25. P. 013118.

$4^{*}$ Письма в ЖТФ, 2017, том 43, вып. 2 